\title{
Numerical Simulation of Barge Impact on a Continuous Girder Bridge and Bridge Damage Detection
}

\section{Yanyan Sha* and Hong Hao}

School of Civil and Resource Engineering, the University of Western Australia 35 Stirling Highway, Crawley WA 6009, Australia

(Received date 9 May 2012; Accepted date 17 Jan 2013)

\begin{abstract}
Vessel collisions on bridge piers have been frequently reported. As many bridges are vital in transportation networks and serve as lifelines, bridge damage might leads to catastrophic consequences to life and economy. Therefore it is of great importance to protect bridge structures, especially bridge piers, against vessel impacts. Many researches have been conducted to predict vessel impact loads on bridge piers, and to design bridge piers or additional protective structures to resist such impact loads. Studies on assessing the bridge conditions after a vessel impact are, however, very limited. Current practice basically uses visual inspections, which not only requires very experienced engineers to perform the inspection in order to obtain creditable assessment, but also is often very difficult to inspect the underwater pier conditions. Therefore it is necessary to develop methods to give efficient, quantitative and reliable assessment of bridge conditions under ambient conditions after a vessel impact. This study explores the feasibility of using vibration measurements to quickly detect bridge conditions after a vessel impact. The study consists of three parts. First, a detailed numerical model of an example bridge structure is developed to calculate the vibrations under ambient hydrodynamic force. Then the model is used to simulate vessel impact on bridge pier and predict the pier damage. The vibration response analysis of the damaged bridge model is performed again in the third step to simulate vibration responses of the damaged bridge under ambient conditions. Using the vibration data obtained before and after vessel impact, the bridge vibration parameters such as vibration frequencies and mode shapes are extracted by using the frequency domain decomposition method. The bridge condition will then be identified through the changes in bridge vibration parameters and compared with the damage observed in the impact simulation. It is found that this method is capable of estimating bridge damage condition after barge impact accident.
\end{abstract}

Key words: Vessel collision; Bridge condition; Pier damage 


\section{INTRODCUTION}

Bridges across waterways are under the threat of accidental vessel impacts during its service life. Statistics show that more than one severe vessel-bridge collision accidents occur each year worldwide [1]. As bridges are vital transportation connections, the influence of bridge damage or even collapse due to vessel collision could be catastrophic. Therefore it is important to protect bridge structure, especially bridge piers, against vessel impact loads. Bridge protection devices are developed to protect bridge piers from vessel collision. However, these devices usually take part of the waterway which narrowed the navigation channel and increase the chance of collision. In addition, designing and building such devices are quite expensive which is not necessarily cost effective. Thus, it is essential to design the bridge pier to resist the collision load by itself. In the past decades, some experimental and numerical studies on vessel-bridge impact have been conducted. Minorsky [2] and Woision [3] carried out scaled ship-ship collision tests and proposed empirical formulas to calculate impact force. Meir-Donberg [4] investigated barge collision through barge impact tests. Based on his research results, the American Association of State Highway and Transportation Officials (AASHTO) published the Guide Specification and Commentary for Vessel Collision Design of Highway Bridge [5]. In addition to these scaled tests, Consolazio et al [6] conducted in site barge pier impact tests in the St. George Island Causeway Bridge in 2004. Their research indicated AASHTO specification which neglects dynamic effects may give inaccurate predictions on the collision load. Numerical studies have also been carried out by a group of researchers. Those numerical investigations [7-9] studied relationships between impact force and barge crush depth. Sha and Hao [10, 11] calculated impact force time history and pier top displacement considering bridge superstructure effect, as well as interaction between barge and pier structures.

Most of the previous works focus on the prediction of the impact load during the collision. Simplified impact models and empirical equations are proposed for calculation the maximum and the equivalent impact forces. Although these efforts give useful information for new bridge designs, they cannot give a quick assessment of bridge conditions after a vessel impact accident. As it is also important to quickly identify the conditions of bridges after vessel collision accidents to minimize the service interruption, it is essential to develop some efficient and reliable ways to assess the conditions of the bridge that has suffered vessel impact. In current practice, visual inspection is the common way employed to assess the integrity of the structure after an accident. Visual inspection method is straightforward but it requires very experienced engineers to perform the inspection on site in order to obtain creditable assessment. For a bridge structure suffered vessel impact, it is very difficult to visually inspect the underwater pier conditions. Moreover, this method can only give general condition of the structure, a more efficient and quantitative assessment method should be adopted.

The dynamic vibration testing is a method which is more and more widely used in the area of structural health monitoring of bridge structures. Through dynamic vibration testing, modal parameters (natural frequencies, damping ratios and mode shapes) can be extracted and employed to detect and locate damage in bridge structures. Forced vibration test and ambient vibration test are two common types of dynamic testing methods. In the forced vibration test, drop weight or shaker is commonly utilized to excite the structure, but this method has a disadvantage of the traffic shut down during the experiment. Ambient vibration test, however, does not interrupt traffic and only uses wave or wind load as natural excitations. The limitation of ambient vibration test is that it is difficult to measure the input 
excitations. Thus, output-only modal identification method need be utilized to identify the structure condition. The output-only method is relatively inexpensive since no excitation equipment is needed. To assess the condition of a bridge suffered vessel impact, output-only method is probably a more suitable choice to avoid causing further damage to the bridge structure.

With only the output data recorded from accelerometers distributed on the structure, the modal parameters can be identified through various methods. Frequency domain decomposition method [12] is an effective method to extract the modal parameters subjected to stochastic excitation. This method can identify close modes with high accuracy even in the case of highly contaminated signals. With this technique, natural frequencies and mode shapes of the structure can be identified. A lot of research works have been done to detect structure damage using shifts in natural frequencies. Salawu [13] and Doebling et al. [14] reviewed literatures on the change of frequencies to identify damage in the structures. The natural frequencies are easy to measure and can be used for damage diagnosis. It should be noted that the natural frequencies can only identify damages without spatial information, except at the higher modal frequencies which contains information of local response. Mode shape and modal assurance criterion can also be used to determine the level of correlation between the undamaged and damaged modes [15]. Compared with natural frequencies, the changes in mode shapes are more sensitive to damage. However, local damage may not have significant influence on the change of lower modes of large structures [16]. A large number of research papers have been published in the literature to use vibration measurements to identify bridge conditions. However, no study specifically devotes to using vibration measurements for a quick bridge condition assessment after a vessel collision accident.

In this paper, the feasibility of using vibration measurement under ambient condition to assess the bridge conditions after a vessel impact accident is explored. A three span continuous girder bridge is used as the example. To achieve the objective of this study, the analysis procedure is divided into three steps as follows. In the first step, ambient hydrodynamic load is applied to bridge piers to excite the bridge structure. The acceleration responses are calculated, representing acceleration data captured by accelerometers distributed on the bridge deck. These data represent the measurements before the accident. In the following step, barge impact load is applied to the bridge pier. Impact forces, bridge responses and pier damage conditions are calculated in this stage. To cover possible impact cases, three impact conditions representing low, medium and high impact energy are considered in this step. In the last step, after the bridge vibration in the second step simulation stops, the same ambient hydrodynamic force is applied to the bridge pier to calculate acceleration responses. The acceleration responses calculated before and after vessel impact are analyzed to derive the modal parameters of the bridge model. The changes in the bridge modal parameters are analyzed to identify bridge conditions. The identified bridge conditions are compared with the damage observed in the impact simulation. The numerical results obtained in this study demonstrate the feasibility of using vibration measurement to quickly assess bridge conditions should a vessel impact accident occur. In practice, measurement of bridge vibration responses to ambient hydrodynamic forces can be incorporated in the periodic bridge condition assessment in bridge management program. These measured data representing those before the impact can be used as the references for bridge damage identification after a vessel collision accident. 


\section{FINITE ELEMENT MODEL OF BARGE AND BRIDGE}

\subsection{BRIDGE AND BARGE MODEL DESCRIPTION}

Without losing generality, a $120 \mathrm{~m}(30 \mathrm{~m}+60 \mathrm{~m}+30 \mathrm{~m})$ three-span continuous girder bridge is selected to perform barge impact simulation in this study. Figure 1 shows the elevation view of the bridge. As the barge is assumed to only collide into pier 2, very detailed modeling with fine mesh is only applied to this pier to save modeling and computational cost. The pier has a circular shape with a diameter of $2.8 \mathrm{~m}$ and height of $25.2 \mathrm{~m}$. The pier is assumed to have a reinforcement cover depth of $50 \mathrm{~mm}$. As show in Figure 2, longitudinal steel

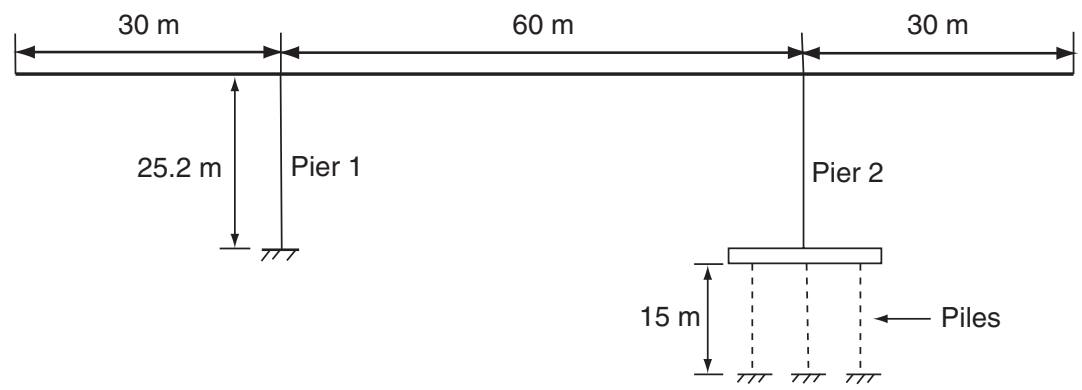

Figure 1. Elevation view of the three-span bridge

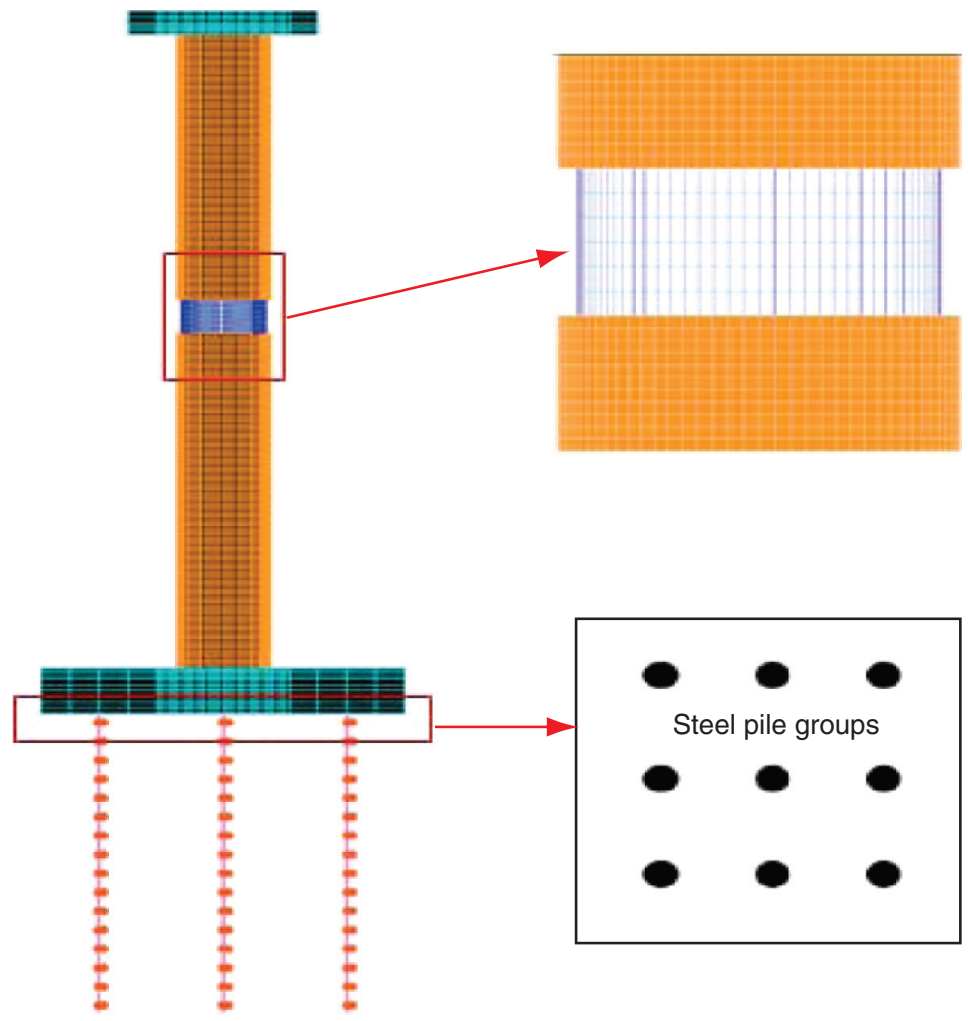

Figure 2. FE mesh of pier column including reinforcement bars and pile foundation 
reinforcements with a diameter of $30 \mathrm{~mm}$ are spaced at a distance of $300 \mathrm{~mm}$ and stirrups of diameter $20 \mathrm{~mm}$ are spaced at $200 \mathrm{~mm}$ along the pier height. Perfect bond assumption is adopted in this study to model the connections between the steel reinforcements and concrete.

The pile foundations and soil-structure interaction effects are also considered in the finite element model. The pile foundation considered includes a pile cap supported by nine $15 \mathrm{~m}$ long steel piles as shown in Figure 2. The effect of surrounding soil is modeled by incorporating nonlinear spring elements to the piles. Nonlinear discrete spring elements are used to model the soil-pile interaction and beam elements are used to represent the steel piles. As shown in Figure 2, the soil springs are placed along the pile at a distance of $600 \mathrm{~mm}$ at each of the pile nodes. Two transverse springs are placed perpendicularly to model the constraints of surrounding soil. Nonlinear loading and unloading curves representing force-deformation data pairs defined for soil springs in [17] are adopted in this study. The bridge deck and pier 1 are simply modeled by beam elements to save computational time as no major deformation is expected to occur in these parts.

The jumbo hopper barge which is widely used in inland waterways is selected as the barge model in the current study [5]. The length and width of the barge are $59.4 \mathrm{~m}$ and $10.6 \mathrm{~m}$ respectively. The barge bow area is modeled in details with a minimum mesh size of $50 \mathrm{~mm}$ as this part will directly impact on the pier and large deformation is expected in this part. It is modeled by steel outer plates and internal trusses to represent the actual stiffness of the structure. The barge hopper is simply modeled to represent the actual mass distribution of the barge. The modeling details of the barge model are described in Sha and Hao [10].

\subsection{ELEMENT AND CONTACT}

A three dimensional finite element model of the bridge is developed in the current study. The concrete and steel reinforcement in pier 2 are modeled by solid and beam elements, respectively. A mesh size of $50 \mathrm{~mm}$ is used. The bridge deck and pier 1 are modeled by beam element with coarse mesh of $0.5 \mathrm{~m}$ as no large deformations are expected in this area. The FE model of the barge is shown in Figure 3. Shell and beam elements are used to model the steel plates and trusses in the barge bow, respectively. A fine mesh is defined for the front part of the barge bow which will directly impact on pier 2. The hopper part of the barge which only contributes to mass distribution is simply model by solid element with coarse mesh size. Mesh convergence test is performed and proves the current mesh size is appropriate for the numerical simulation.

The treatment of sliding and impact along interfaces of different elements is an important issue in large deformation finite element modeling. Two contact algorithms, namely CONTACT_AUTOMATIC_SURFACE_TO_SURFACE (ASTS) and CONTACT_ AUTOMATIC_SINGLE_SURFACE (ASSC) in the nonlinear finite element code LSDYNA are employed in the simulations. The contact algorithm ASTS is defined for the contact between the barge bow and pier to avoid penetration at the interface with different mesh sizes. The dynamic and static Coulomb friction values are both set to 0.3. Large deformation may also occur between the outer shells and internal trusses in barge bow area, ASSC with a contact friction value of 0.21 is defined for these two parts. By implementing ASSC, it will only cause minor increases in computational time, and no contact or target surface definitions are required. 


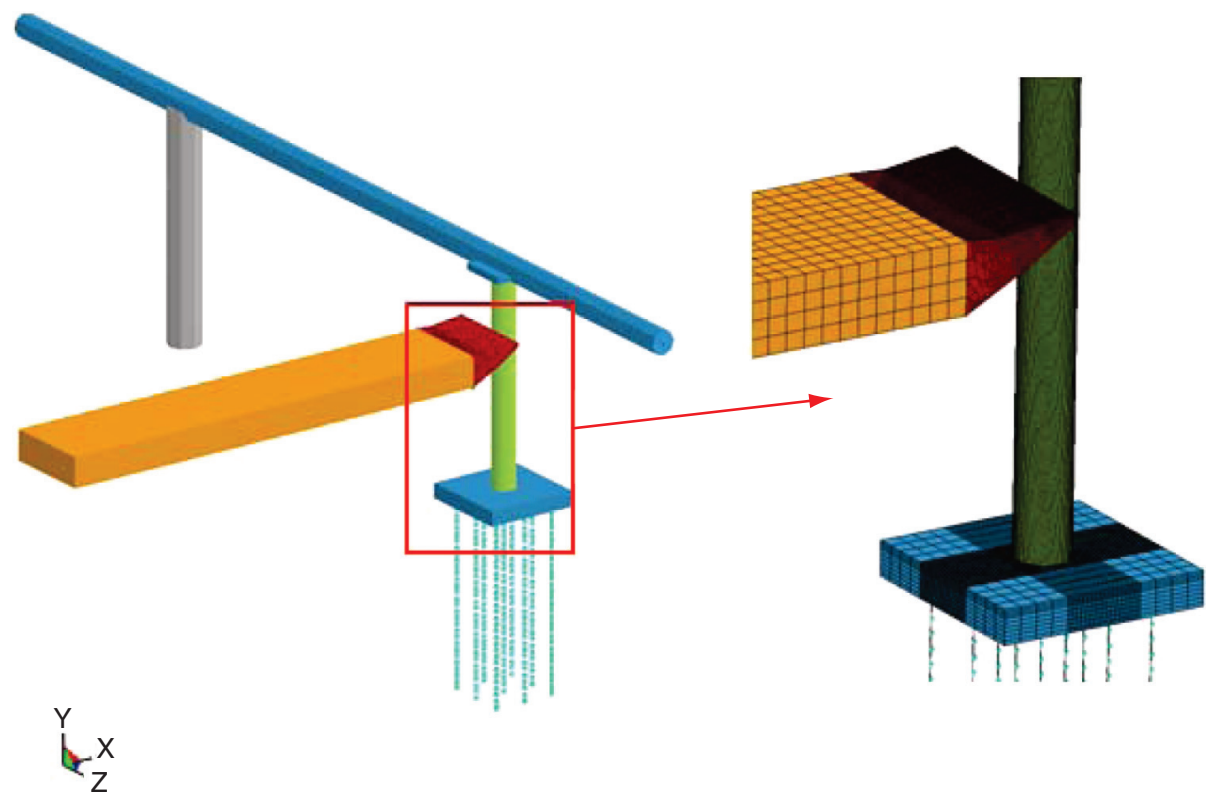

Figure 3. Finite element models of barge and bridge

\subsection{MATERIAL MODEL}

Pier 2 is modeled in detail by nonlinear material models. The concrete material of the pier is modeled by MAT_CONCRETE_DAMAGE_REL3. This material is able to model the dynamic behavior of concrete under impact loading [18]. The advantage of this material is the automatic generation of material parameters with only the input of density, Poisson's ratio and unconfined compressive strength of the concrete. During the impact, failed concrete elements which do not further contribute to resist the impact load are eliminated in order to avoid computation overflow. Since the concrete material model does not allow failure and erosion of element, the card MAT_ADD_EROSION is utilized to eliminate the failed elements. In the present study, the elements will be deleted when the principal strain reaches 0.1 . Steel reinforcements in pier 2 are modeled by MAT_PIECEWISE_LINEAR_PLASTICITY. This elasto-plastic model with user-defined stress strain curve and stain rate dependency is commonly used by researchers. Smeared concrete is utilized in modeling the pile cap and nine steel piles are connected to the bottom of the cap. The bridge deck and pier 1 are modeled by elastic materials. The material model MAT_PLASTIC_KINEMATIC is employed to model the outer shell and internal truss of the barge bow. It is a cost effective model for isotropic and kinematic hardening plasticity. The rear part of the barge is modeled by elastic material. The parameters of the material models are tabulated in Table 1. The finite element models of the whole impact system are shown in Figure 3. This FE model was calibrated with the experimental results by Sha and Hao [11]. The numerical results showed good agreement with the impact tests, indicating the current FE model can give good predictions of bridge and barge responses and damage owing to barge collision. More information for impact tests and model calibration can be found in [11]. 
Table 1. Material parameters for the bridge and the barge

\begin{tabular}{lccc} 
Material & LS-DYNA Model & Input Parameter & Magnitude \\
\hline Steel & *MAT_PIECEWISE_LINEAR_ & Density & $7700 \mathrm{~kg} / \mathrm{m} 3$ \\
reinforcement & PLASTICITY & Young's modulus & $200 \mathrm{GPa}$ \\
& & Poisson's ratio & 0.3 \\
& & Yield stress & $550 \mathrm{MPa}$ \\
& & Tangent modulus & $1600 \mathrm{MPa}$ \\
& & Fraction strain & 0.35 \\
\hline Concrete & Mass density & $2400 \mathrm{~kg} / \mathrm{m} 3$ \\
(Pier 2) & *MAT_CONCRETE_ & Compressive strength & $28.3 \mathrm{MPa}$ \\
\hline Smeared & DAMAGE_72Rel3 & Mass density & $2180 \mathrm{~kg} / \mathrm{m} 3$ \\
concrete & *MAT_ELASTIC & Young's modulus & $30 \mathrm{GPa}$ \\
(Pier 1) & & Poisson's ratio & 0.2 \\
\hline Steel Pile & & Mass density & $7830 \mathrm{~kg} / \mathrm{m} 3$ \\
& *MAT_RIGID & Young's modulus & $207 \mathrm{GPa}$ \\
& & Poisson's ratio & 0.28 \\
\hline Smeared Concrete & *MAT_ELASTIC & Mass density & $2564 \mathrm{~kg} / \mathrm{m} 3$ \\
(Bridge deck) & & Young's modulus & $30 \mathrm{GPa}$ \\
& & Poisson's ratio & 0.2 \\
\hline Steel & Mass density & $7865 \mathrm{~kg} / \mathrm{m} 3$ \\
(Barge bow) & Young's modulus & $207 \mathrm{GPa}$ \\
& KINEMATIC & Poisson's ratio & 0.27 \\
& & Yield stress & $310 \mathrm{MPa}$ \\
& & Failure strain & 0.35 \\
& & C & 40 \\
& & P & 5 \\
\hline
\end{tabular}

\section{BARGE IMPACT AND AMBIENT TESTING}

\subsection{HYDRODYNAMIC LOADING}

The piers will subject to ambient hydrodynamic loads of various forms and intensities during its service life. The hydrodynamic loads include hydrostatic pressure, buoyancy force, drag force and so on. In this study, only the hydrodynamic drag force is applied to both piers. According to the Australian Standard AS 5100.2 [19], the fluid forces on the bridge pier are dependent on the pier shape, the water velocity and the direction of the water flow. The hydrodynamic drag force in the direction of a steady flow can be calculated by the following equation:

$$
F_{D}=\frac{1}{2} \rho C_{D} A u_{p}^{2}
$$

where $\rho$ is the water density and $C_{D}$ is the drag coefficient. $A$ and $u_{p}$ are projected area normal to the direction of the flow and water velocity, respectively. The drag coefficient depends on the pier shape and is taken as 1 for circular pier and the velocity of water is assumed as $2 \mathrm{~m} / \mathrm{s}$ in this study. The calculated hydrodynamic load is smeared with a white noise of 10 percent and then used as the ambient loading to calculate the bridge response. 


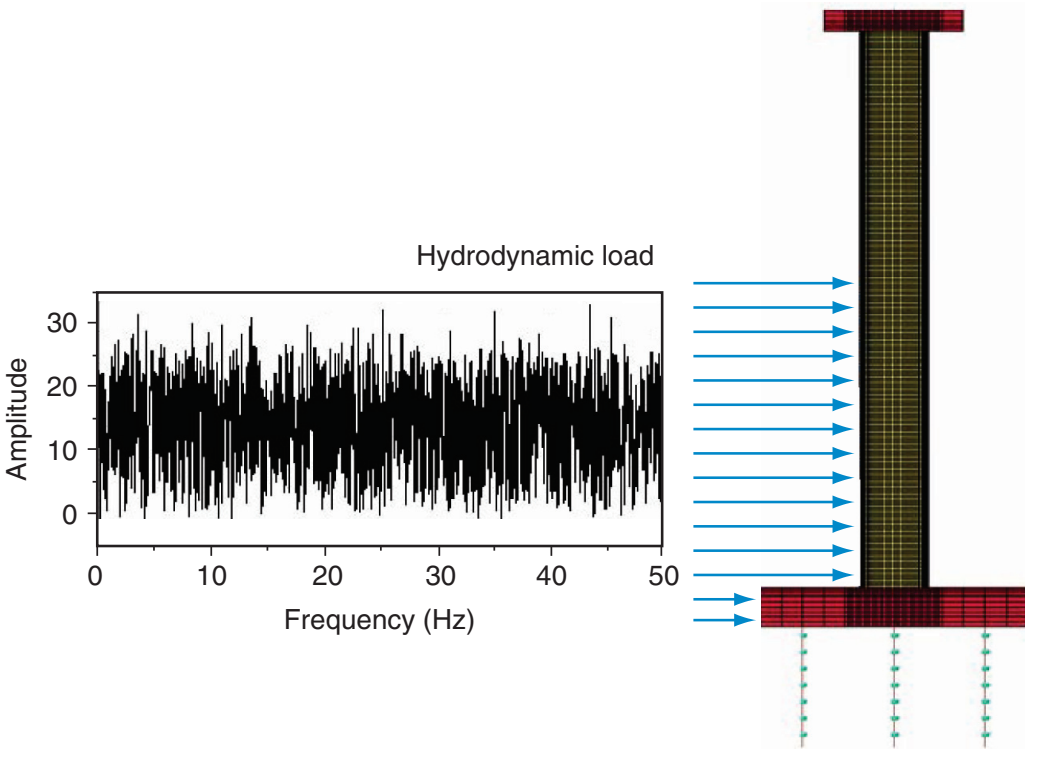

Figure 4. Hydrodynamic load applied on the piers

The hydrodynamic load is applied to both piers under the water level and the frequency response spectrum of the ambient hydrodynamic loading is also shown in Figure 4. It should be noted that in reality the hydrodynamic force is not uniformly distributed along the water depth. In this study, however, a uniform loading distribution is assumed. This is because the main objective of this study is to calculate the bridge damage induced by barge impact, and use vibration-based method to identify the damage. Simulating accurate hydrodynamic load acting on bridge piers is beyond the scope of this study.

\subsection{AMBIENT BRIDGE VIBRATION BEFORE BARGE IMPACT}

In the first stage, bridge responses to ambient hydrodynamic force, simulating ambient vibration testing, are calculated before barge collision to obtain the undamaged bridge condition. The hydrodynamic load described above is applied to the front face of two piers under the waterline (see Figure 4). The bridge acceleration responses under the excitation are then collected by nine accelerometers distributed on the bridge deck. The arrangement of accelerometers is shown in Figure 5. Bridge responses under hydrodynamic loading are recorded for 32s with a sample frequency of $50 \mathrm{~Hz}$. Figure 6 shows the time history of a typical horizontal acceleration data at sensor location 7.

\subsection{BARGE IMPACT}

With the initial bridge conditions obtain by ambient excitation, the Jumbo Hopper barge impact on bridge pier is then simulated and the induced bridge damage is estimated. As bridge structures may be subjected to barge collisions with various payload and speed which will result in different extents of damage conditions. Three typical cases which represent low, medium and high energy impact scenarios are simulated in the study (see Table 2). Barge with a mass of 952 ton travelling at $3.09 \mathrm{~m} / \mathrm{s}$ is considered as the low energy impact case while a 1723 ton barge impacting at $4.11 \mathrm{~m} / \mathrm{s}$ is selected as the medium impact energy case. In the high energy impact case, the impacting barge with a total mass of 2125 ton is assumed colliding into the bridge pier at a velocity of $6.17 \mathrm{~m} / \mathrm{s}$. 


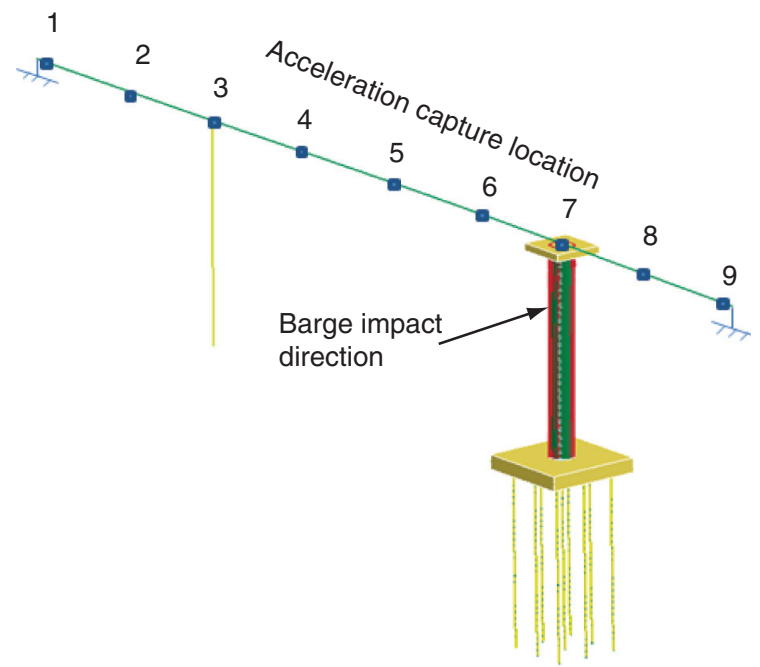

Figure 5. Bridge deck accelerometer arrangement

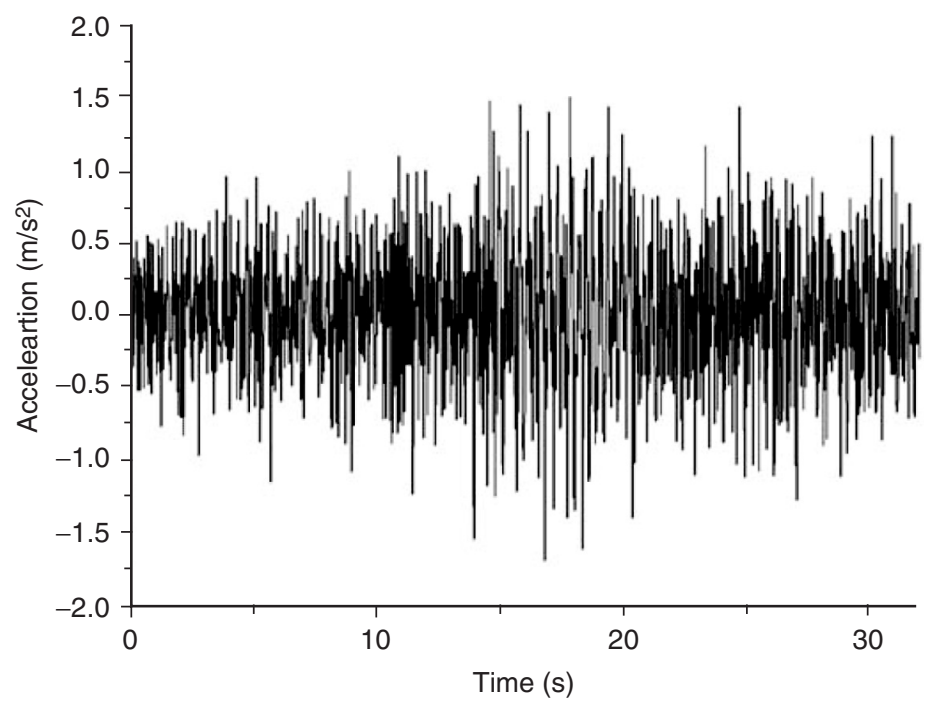

Figure 6. Time history of horizontal acceleration at sensor location 7 before impact

Barge-bridge impact force and bridge response can vary greatly when the impact energy changes. Here, the peak impact force, the impact duration and the maximum pier displacement of these impact cases are calculated and also given in Table 2. As can be seen from the table, the peak impact forces of low and medium impact cases are 7.26 MN and 7.70 MN, respectively. However, the maximum impact force increases to $9.26 \mathrm{MN}$ in the high energy impact case. It is because the total impact force is governed by the contact area between the barge and impacted pier besides the impact energy. When the impact energy is small and there is no significant barge bow deformation, the barge and the pier experiences basically point contact only because of the circular shape of the pier. Therefore the total 
Table 2. Different cases studied

\begin{tabular}{lcccc} 
& Parameters & $\begin{array}{c}\text { Low } \\
\text { energy }\end{array}$ & $\begin{array}{c}\text { Medium } \\
\text { energy }\end{array}$ & $\begin{array}{c}\text { High } \\
\text { energy }\end{array}$ \\
\hline Impact characteristic & Barge mass (ton) & 952 & 1723 & 2125 \\
\cline { 2 - 5 } & Barge impact velocity (m/s) & 3.09 & 4.11 & 6.17 \\
\cline { 2 - 5 } & Barge impact energy (MJ) & 4.54 & 14.55 & 40.45 \\
\hline Impact force and pier & Peak impact force (MN) & 7.26 & 7.70 & 9.26 \\
\cline { 2 - 5 } response & Impact duration (s) & 1.035 & 2.716 & 3.1275 \\
\cline { 2 - 5 } & Maximum pier displacement (m) & 0.211 & 0.208 & 0.448 \\
\hline
\end{tabular}

(a)

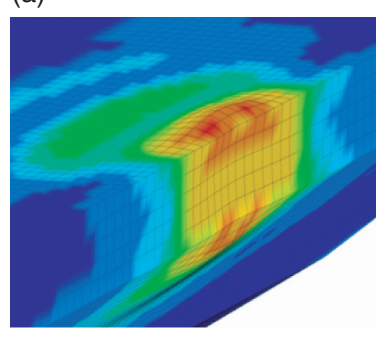

(b)

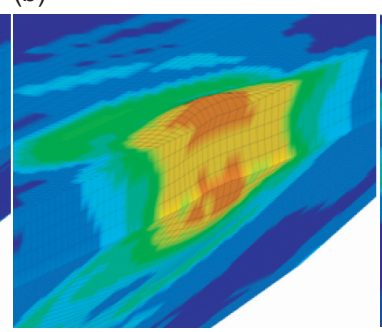

(c)

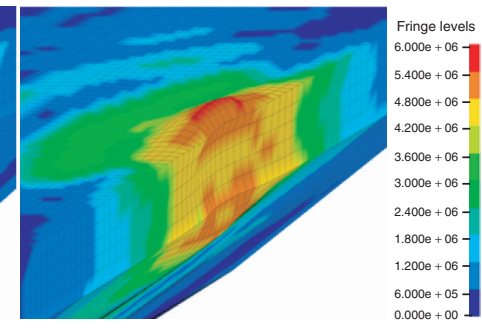

Figure 7. Barge bow stress contour at the time instance of maximum impact force. (a) Low energy impact; (b) Medium energy impact; (c) High energy impact

impact force acting on pier is relatively small and concentrating at a single point. When large deformation of barge bow occurs, more barge bow will be in contact with the circular pier. The total impact force acting on the pier increases. As can be seen in the stress contour in Figure 7 which shows the stress distribution at the impact instant in barge bow, the contact areas in medium impact case is slightly larger than the low energy impact case because of the larger deformation of the barge bow. This causes an increment of six per cent in peak impact force. When the impact energy increases to $40.45 \mathrm{MJ}$ in the high energy impact case, more shell elements in the barge bow are involved in the collision which leads to a much larger total impact force. The impact time duration also increases with the impact energy and the duration of the high energy impact case is about three times of the low energy case because of again more significant deformations in barge bow. This indicates the interaction, i.e., the energy exchange between the barge and the pier is more intensive when the impact energy is larger.

Generally the maximum pier displacement increases gradually with the increment of impact energy. However, the maximum pier displacement in low energy impact case is $0.211 \mathrm{~m}$, slightly larger than that of the medium energy impact case $(0.208 \mathrm{~m})$. This is because the internal energy in the medium impact case is smaller than the low impact case which results in a relatively smaller pier displacement (see Figure 8), but the eroded energy in the medium impact case is large than the low energy impact case which means the pier suffers severer damage. In other words, the medium energy impact case causes more localized damage to the pier, which absorbs more impact energy and results in slightly smaller pier overall displacement response. The maximum pier displacement increases to $0.448 \mathrm{~m}$ in the high 
(a)

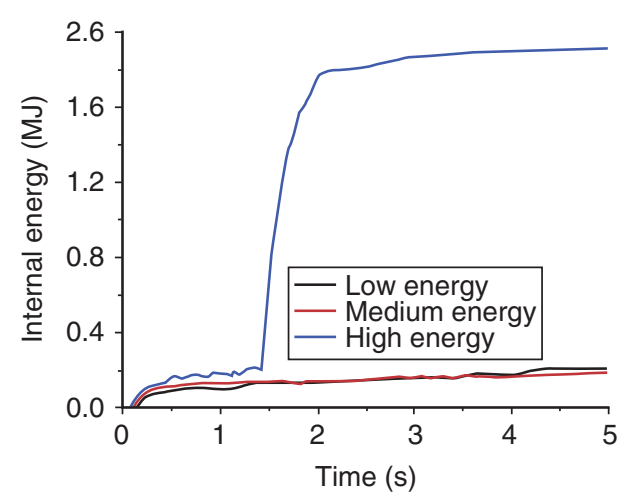

(b)

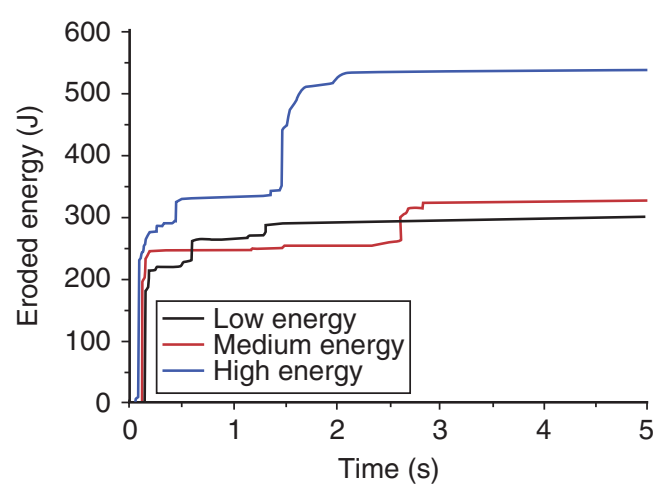

Figure 8. Pier energy in various cases. (a) internal energy; (b) eroded energy

energy impact. As shown in Figure 8, the pier has much larger internal energy and eroded energy in this case which results in a larger pier response and structural damage. It should be noted that the sudden increase at around $1.45 \mathrm{~s}$ is because the barge hopper collides with the pier when the barge bow is totally crushed at this instant. Figure 9 illustrates the deformation and damage of the impacted pier under various impact energies. It can be observed from the figure that the plastic deformation and damage of the pier increases as the impact energy increases. In the low energy impact case, the impacted bridge pier can almost restore its original position with minor concrete elements failure on the pier concrete cover. Plastic deformation and damage occur in the medium energy impact case but no significant damage can be viewed from visual inspection. Severe plastic deformation can be observed in high energy impact case and large amount of concrete elements fail in the pier.

\subsection{AMBIENT BRIDGE VIBRATION AFTER BARGE IMPACT}

After the barge impact, the restart technique in LS-DYNA is utilized to conduct response analysis of the damaged bridge model to ambient hydrodynamic force again, simulating a vibration test after a barge impact accident. The keyword STRESS_INITIALIZATION is LS-DYNA is used to initialize the stress state of the whole bridge after barge impact. After the stress state is initiated, the same hydrodynamic load is then applied to the same location 
(a)

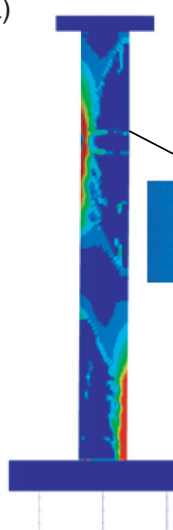

(b)

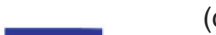

(c)

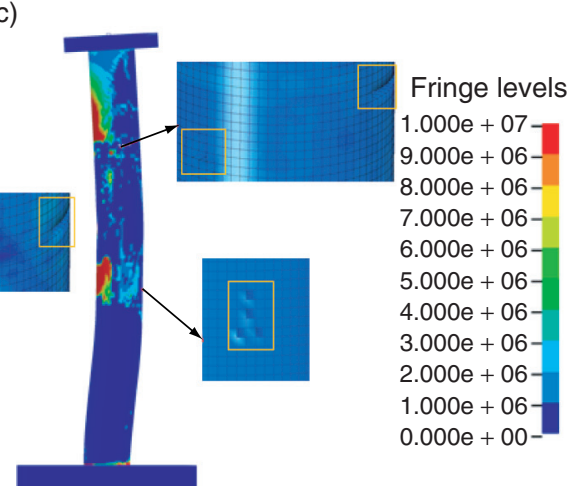

Figure 9. Stress contour of the impacted pier. (a) low energy impact; (b) medium energy impact; (c) high energy impact

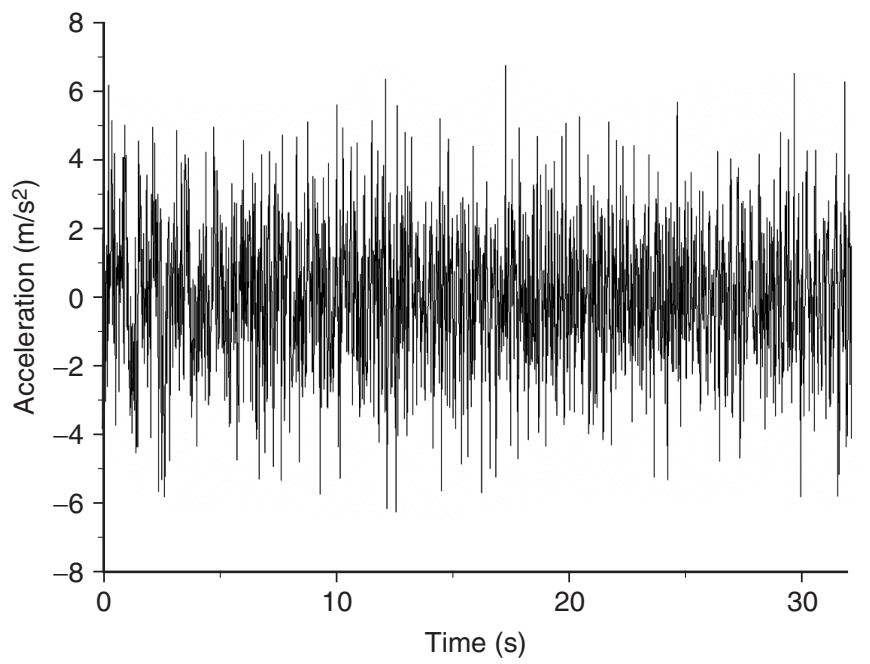

Figure 10. Time history of horizontal acceleration at sensor location 7 after impact

of both bridge piers. The acceleration response time histories at the nodes defined in Figure 5 are calculated. Using these acceleration time histories, modal parameters after impact can be extracted and compared with the modal parameters before barge impact. Bridge damage condition can be predicted with the identified modal data. A typical acceleration signal collected after barge collision is shown in Figure 10.

\section{MODAL DATA EXTRACTION AND DAMAGE CORRELATION}

The presence of damage in a structure causes changes in modal parameters. Thus, the change of modal parameters such as the natural frequencies and mode shapes can be used as a data feature for detecting structure damages [13]. To obtain these modal parameters, the 
acceleration data of the structure should be collected and analyzed by modal identification method. In this paper, frequency domain decomposition method is used to extract the modal parameters of the bridge.

\subsection{FREQUENCY DOMAIN DECOMPOSITION}

The simplest method to predict modal parameters subjected to stochastic excitation is the peak-picking method [20]. The natural frequencies are identified by the peaks of a frequency spectrum and the mode shapes by a decomposition of the output power spectrum at these natural frequencies. However, the selection of natural frequencies is subjective in this method. This approach is unable to estimate damping or identify modal parameters of closely spaced modes. A variant of the peak-picking method known as frequency domain decomposition (FDD) method was proposed by Brincker, et al. [12]. Instead of using the spectral matrix directly for the classical peak-picking approach, the spectral matrix is decomposed applying the singular value decomposition (SVD) into a set of auto spectral density functions, each corresponding to a single degree of freedom system. The singular vectors are interpreted as mode shape vectors and the natural frequencies are estimated by taking each single degree of freedom auto spectral density function back to time domain by inverse discrete Fourier transform.

It is beyond the scope of this paper to explain in detail the FDD method. Only the main ideas of the method are described herein. In the FDD method, the first step is to estimate the power spectral density matrix and the SVD of the spectral matrix at each frequency. The next step is the inspection of the curves representing the singular values to identify the resonant frequencies and estimate the corresponding mode shape using the information contained in the singular vectors of the SVD. The SVD of the spectral matrix at discrete frequencies $\omega=\omega_{i}$ is given by

$$
G_{y y}\left(j \omega_{i}\right)=U_{i} S_{i} U_{i}^{H}
$$

where the matrix $U_{i}$ is a unitary matrix holding the singular vectors $u_{i j}$, and $S_{i}$ is a diagonal matrix holding the scalar singular values $S_{i j}$. The general multi-degree of freedom (DOF) system can be transformed to the single DOF system close to its natural frequencies by means of SVD. The mode shape can be estimated as the first column vector of the unitary matrix of $U_{i}$, since the first singular value may include the structural mode close to its natural frequencies. However, in the closely spaced modes, the peak of the largest singular values at one natural frequency indicates the structural mode, and an adjacent second singular value may indicate the close mode. This method operates well in the case where the loading is white noise and the structure is lightly damped. Even these assumption are not satisfied, the modes can still be identified with good accuracy.

\subsection{RESULTS AND DISCUSSIONS 4.2.1. Natural frequency}

The amount of literature that uses the change of natural frequencies as a feature for detecting structural damage is quite large. It is well known that the changes in structural properties cause frequency shifts in the structures, and by comparing the shifts of natural frequencies, the structural damage can be quickly and reliably identified.

In this study, to investigate the influence of measurement noise in practice on vibration parameters extraction, two noise levels, i.e. no noise and $10 \%$ noise, are smeared in the calculated acceleration data. The noise-free and noise-polluted data are then analyzed 
Table 3. Bridge natural frequencies before and after various impact cases

Cases

\begin{tabular}{|c|c|c|c|c|c|c|c|}
\hline \multirow{7}{*}{$\begin{array}{l}\text { No } \\
\text { noise }\end{array}$} & Before impact & Frequency $(\mathrm{Hz})$ & 0.812 & 1.53 & 2.561 & 3.467 & 6.652 \\
\hline & 952 ton $3.09 \mathrm{~m} / \mathrm{s}$ & Frequency $(\mathrm{Hz})$ & 0.7324 & 1.5 & 2.5 & 3.5 & 6.563 \\
\hline & & Percent change & $-9.80 \%$ & $-1.96 \%$ & $-2.38 \%$ & $0.95 \%$ & $-1.34 \%$ \\
\hline & 1723 ton, $4.11 \mathrm{~m} / \mathrm{s}$ & Frequency $(\mathrm{Hz})$ & 0.7188 & 1.531 & 2.5 & 3.438 & 6.531 \\
\hline & & Percent change & $-2.22 \%$ & $0.1 \%$ & $-2.38 \%$ & $-0.84 \%$ & $-1.82 \%$ \\
\hline & $17 \mathrm{~m} / \mathrm{s}$ & Frequency $(\mathrm{Hz})$ & 0.6838 & 1.496 & 2.479 & 3.462 & 6.453 \\
\hline & & Perc & $-5.79 \%$ & $-2.22 \%$ & $-3.20 \%$ & $-0.14 \%$ & $-2.99 \%$ \\
\hline \multirow{6}{*}{$\begin{array}{l}10 \% \\
\text { noise }\end{array}$} & 952 ton $3.09 \mathrm{~m} / \mathrm{s}$ & Frequency $(\mathrm{Hz})$ & 0.7324 & 1.514 & 2.515 & 3.491 & 6.592 \\
\hline & & Percent change & $-9.80 \%$ & $-1.05 \%$ & $-1.80 \%$ & $0.69 \%$ & $-0.90 \%$ \\
\hline & 1723 ton, $4.11 \mathrm{~m} / \mathrm{s}$ & Frequency $(\mathrm{Hz})$ & 0.7188 & 1.514 & 2.49 & 3.467 & 6.543 \\
\hline & & Percent change & $-2.22 \%$ & $-1.05 \%$ & $-2.77 \%$ & $0.00 \%$ & $-1.64 \%$ \\
\hline & 2025 ton $6.17 \mathrm{~m} / \mathrm{s}$ & Frequency $(\mathrm{Hz})$ & 0.6836 & 1.5 & 2.49 & 3.467 & 6.47 \\
\hline & & Percent change & $-5.81 \%$ & $-1.96 \%$ & $-2.77 \%$ & $0.00 \%$ & $-2.74 \%$ \\
\hline
\end{tabular}

with the frequency domain decomposition method. The first five natural frequencies of the bridge before and after barge impact are identified. The results are given in Table 3 . It shows that the first five natural frequencies generally decrease with the barge impact energy as severer damage is expected. The natural frequency of the first horizontal bending mode dropped significantly after barge collision which indicated damages occurred in the bridge structure. However, the changes of natural frequencies are less prominent in mode $2-5$. In fact, slight increase in some modal frequencies is observed when the impact energy is low and intermediate. This is because under relatively low impact energy, the induced damage is mainly local at the impact location. This local damage might alter the high vibration modes, in this case mainly mode 4 , which results in slight increase in modal frequency. Nonetheless, vibration frequency of all modes decreases when the severer damage occurs. These results indicate that vibration measurement can be used to quickly assess damage existence in the bridge after a barge impact accident.

\subsubsection{Mode shape}

Although the frequency shift before and after impact can indicate the occurrence of damage in the bridge structure, it cannot give spatially information of the damage. The changes of bending mode shapes, however, can predict both the existence and the location of damage in the structure. In this study, the first three bending mode shapes before and after barge collision are extracted from the vibration accelerations and shown in Figures 11-13. It should be noted that those mode shapes are extracted from the noise-free data. The mode shapes from noise-polluted data are also extracted but are not shown here because they are very similar to those shown in Figures 11-13. As shown after barge collision, damages in the pier induce clear changes in the mode shapes. These mode shape changes tend to be more significant with the increase of the modes. It can be viewed that the largest deviations occur in sensor location 7 where the pier was impacted by the barge. It should be noted that the bending modes of the bridge deck in the lateral direction is not symmetric in this study, it is because the different modeling technique used in the two piers. 


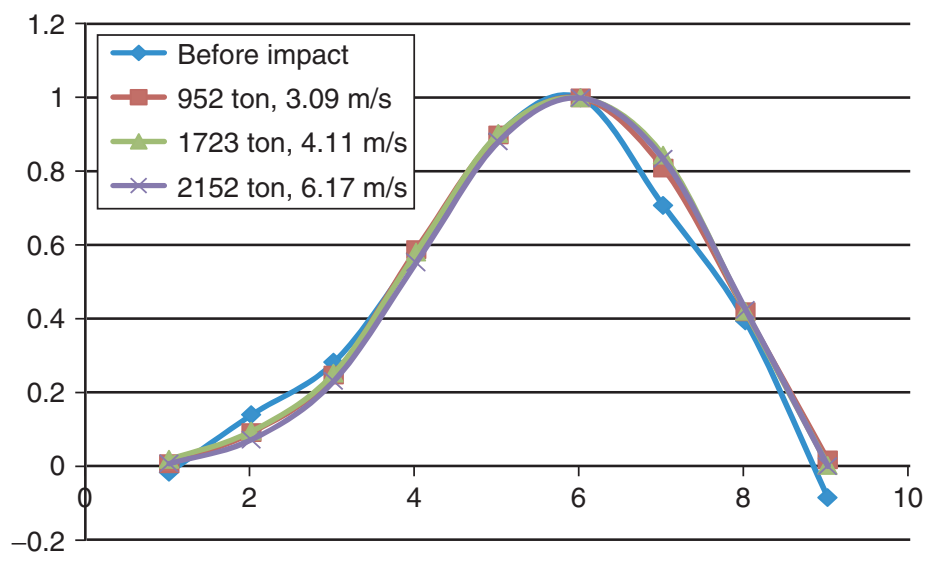

Figure 11. The first horizontal bending mode shapes of the bridge deck before and after barge impact

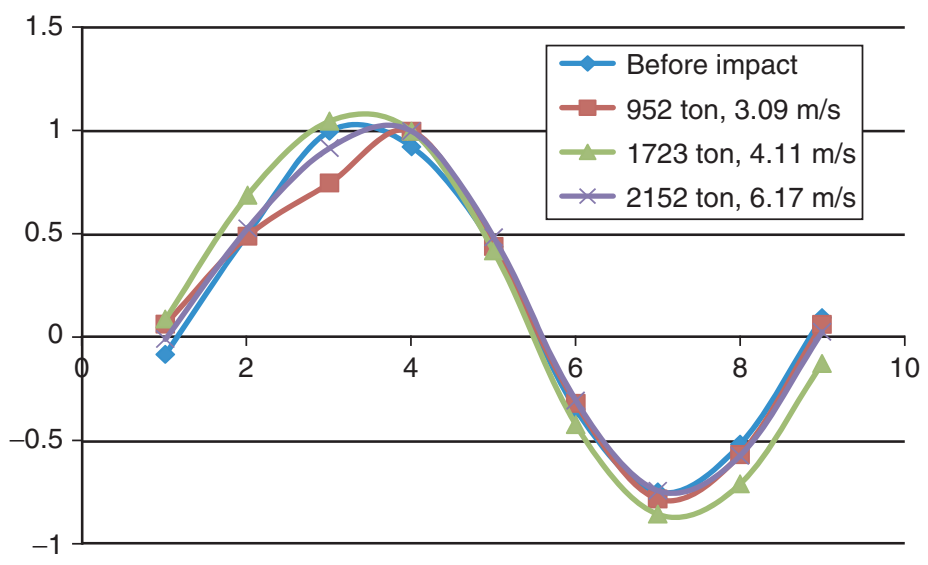

Figure 12. The second horizontal bending mode shapes of the bridge deck before and after barge impact

Modal assurance criterion (MAC) is a simple index to find the correlation between two mode shapes in the damaged and undamaged state [15]. It gives a quantitative value to compare two mode shapes through the following equation:

$$
\operatorname{MAC}\left(\phi_{1}, \phi_{2}\right)=\frac{\left|\phi_{1}^{H} \phi_{2}\right|^{2}}{\left|\phi_{1}^{H} \phi_{1}\right|\left|\phi_{2}^{H} \phi_{2}\right|}
$$

where $H$ denotes complex conjugate and transpose. $\phi_{1}$ and $\phi_{2}$ are the two mode shapes for comparison. MAC values for the first three bending modes before and after barge impact are tabulated in Table 4. It can be observed that the MAC values of the first mode decreases from damage case 1 to damage case 3. However, the differences between MAC values of the first 


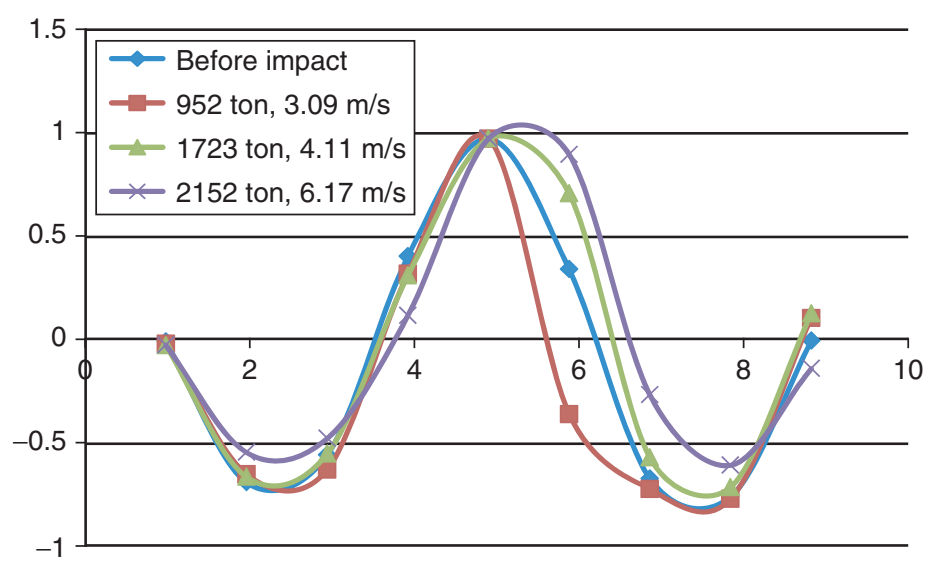

Figure 13. The third horizontal bending mode shapes of the bridge deck before and after barge impact

Table 4. MAC before and after various impact cases

\begin{tabular}{lccc} 
Case & Damage case 1 & Damage case 2 & Damage case 3 \\
\hline Mode 1 & 0.9919 & 0.9907 & 0.9891 \\
\hline Mode 2 & 0.9708 & 0.9682 & 0.9909 \\
\hline Mode 3 & 0.8399 & 0.9449 & 0.7989 \\
\hline
\end{tabular}

mode shape in the three impact cases are not prominent. For the second mode, the reduction of MAC values can be observed in damage case 1 and 2. The MAC values decrease significantly in damage case 1 and 3 for the third mode. These observations indicate the MAC values are able to identify the bridge damage when the second and third bending modes are analyzed together. However, the severity of the damage cannot be accurately predicted based on the MAC values.

\subsubsection{Discussions}

The above numerical simulations demonstrate that the damage existence in bridge pier can be identified through vibration measurements of ambient bridge responses before and after barge impact accident. With damage occurrence, prominent changes in some modal vibration frequencies and MAC values are expected. This allows reliable identifications of damage occurrence. However, using those changes alone are not sufficient to determine the exact damage location and damage severity. Further analysis such as model updating analysis is needed to locate and quantify the damage. This, nevertheless, is beyond the scope of current study.

\section{CONCLUSIONS}

This paper performed numerical simulations of bridge structure damage to barge impact, and investigated the feasibility of using vibration measurements before and after barge impact accident to quickly identify bridge conditions. A detailed finite element model of a threespan continuous bridge and barge structure was developed and utilized to conduct barge impact simulation. Hydrodynamic load with noise was generated as ambient force and 
applied to bridge piers to excite the bridge structure before and after barge impact to simulate vibration measurements. Acceleration responses of the bridge to ambient hydrodynamic force were calculated and used to extract bridge vibration properties. The numerical results demonstrated that damage occurrence in bridge structure induced by barge impact resulted in prominent changes in some modal vibration frequencies and MAC values. These changes could be used to reliably identify damage existence in the bridge. However, further analyses need be carried out to locate and quantify the damage.

\section{ACKNOWLEDGEMENTS}

The first author wishes to thank the China Scholarship Council and The University of Western Australia (UWA) for providing scholarships for him to pursue PhD degree in UWA.

\section{REFERENCES}

[1] Larsen, O. D. Ship Collision With Bridges: the Interaction Between Vessel Traffic and Bridge Structures: IABSE; 1993.

[2] Minorsky, V. U. Analysis of Ship Collisions with Reference to Protection of Nuclear Power Plants. Journal of ship Research. 1959; 3: 1-4.

[3] Woisin, G. The Collision Tests of the GKSS. Jahrbuch der Schiffbautechnischen Gesellschaft. 1976; 70: 465-487.

[4] Meir-Dornberg, K. E. Ship Collisions, Safety Zones, and Loading Assumptions for Structures in Inland Waterways. VDI-Berichte. 1983; 496: 1-9.

[5] AASHTO. Guide Specifications and Commentary for Vessel Collision Design of Highway Bridges: American Association of State Highway and Transportation Officials; 2009.

[6] Consolazio, G., Cook, R., Cowan, D. and Bollmann, H. Assessing Bridge Pier Response to Barge Collision Loads. Zurich, Switzerland: ETH Honggerberg; 2006.

[7] Consolazio, G. R. and Cowan, D. R. Numerically Efficient Dynamic Analysis of Barge Collisions with Bridge Piers. ASCE, Journal of Structural Engineering. 2005; 131: 1256.

[8] Yuan, P. and Harik, I.E. Equivalent barge and flotilla impact forces on bridge piers. Journal of Bridge Engineering. 2010; 15: 523.

[9] Fan, W., Yuan, W., Yang, Z. and Fan, Q. Dynamic demand of bridge structure subjected to vessel impact using simplified interaction model. Journal of Bridge Engineering. 2011; 16: 117.

[10] Sha, Y. and Hao, H. Nonlinear Finite Element Analysis of Barge Collision with a Single Bridge Pier. Engineering Structures. 2012; 41: Pages 63-76.

[11] Sha, Y. and Hao, H. Laboratory tests and numerical simulations of barge impact on circular reinforced concrete piers. Engineering structures. 2013; 46: 593-605.

[12] Brincker, R., Zhang, L. and Andersen, P. Modal Identi_cation from Ambient Responses using Frequency Domain Decomposition. 2000.

[13] Salawu, OS. Detection of structural damage through changes in frequency: a review. Engineering structures. 1997; 19: 718-723.

[14] Doebling, S.W., Farrar, C.R., Prime, M.B. and Shevitz, D.W. Damage identification and health monitoring of structural and mechanical systems from changes in their vibration characteristics: a literature review. Los Alamos National Lab., NM (United States); 1996.

[15] West, W.M. Illustration of the use of modal assurance criterion to detect structural changes in an orbiter test specimen. 1986.

[16] Kim, J.T., Ryu, Y.S., Cho, H.M. and Stubbs, N. Damage identification in beam-type structures: frequencybased method vs mode-shape-based method. Engineering structures. 2003; 25: 57-67.

[17] Consolazio, G. R., Cook, R. A., Biggs, A. E., Cowan, D. R. and Bollmann, H. T. Barge Impact Testing of the St. George Island Causeway Bridge Phase II: Design of Instrumentation Systems. Structures Research Rep. No. 2003; 883. 
[18] Crawford, JE and Malvar, LJ. User's and theoretical manual for K\&C concrete model. Rep. TR-97-53.1, Karagozian \& Case Structural Engineers, Burbank, Calif. 1997.

[19] Standard, A. AS5100. 2-2004, Bridge design — Part 2: design loads. Standards Australia, Sydney (Australia). 2004.

[20] Piersol, AG and Bendat, JS. Engineering applications of correlation and spectral analysis. New York: WileyInterscience; 1993. 